\title{
Estudo de Segurança e Eficácia da Enoxaparina Sódica na Profilaxia e Terapêutica Antitrombótica*
}

\author{
Safety and Efficacy of Sodium Enoxaparin in \\ Anti-Thrombotic Prophylaxis and Treatment.
}

\section{Silvia G Lage ${ }^{1}$, Ricardo T Carvalho ${ }^{1}$, Liliane Kopel', Jaime F Bastos ${ }^{1}$, Marcelo A Ribeiro ${ }^{1}$,} Antonio AP Fagundes Junior ${ }^{1}$, Helia Beatriz N Araujo', Célia C Strunz².

\section{RESUMO}

JUSTIFICATIVA E OBJETIVOS: O uso de heparina de baixo peso molecular (HBPM) na profilaxia ou terapêutica de diversas doenças ou síndromes é de grande importância na prática clínica. O objetivo deste estudo foi avaliar a segurança e a eficácia da enoxaparina sódica, teste (ENOX-T) comparada ao medicamento de referência (ENOX-R).

MÉTODO: Foi realizado estudo prospectivo, aleatório, comparativo, unicêntrico e aberto envolvendo pacientes com indicação de profilaxia ou tratamento antitrombótico. Foram incluídos 100 pacientes divididos em 2 braços: profilático $(n=50)$ e terapêutico $(n=50)$ divididos em 2 grupos para cada braço (grupo 1: ENOX-R e grupo 2: ENOX-T). Analisou-se em cada segmento os dados clínicos e laboratoriais. A atividade do anti-fator Xa foi aferida em três momentos: basal ( $1^{\mathrm{a}}$ avaliação); $1^{\circ}$ ou $2^{\circ}$ dia ( $2^{\mathrm{a}}$ avaliação) e $5^{\circ}$ a $7^{\circ}$ dia ( $3^{\mathrm{a}}$ avaliação). $\mathrm{O}$ ultra-som Doppler dos membros inferiores foi realizado na $2^{\mathrm{a}}$ ou $3^{\mathrm{a}}$ avaliação do braço profilático.

1. Unidade de Terapia Intensiva do Instituto do Coração, HCFMUSP.

2. Laboratório de Análises Clínicas, Instituto do Coração, HC FMUSP.

*Recebido da Unidade de Terapia Intensiva do Instituto do Coração do Hospital das Clínicas da Faculdade de Medicina da Universidade de São Paulo (HC-FMUSP), São Paulo, SP

Apresentado em 25 de novembro de 2006

Aceito para publicação em 06 de fevereiro de 2007

Endereço para correspondência:

Prof $^{a}$. Dr ${ }^{\mathrm{a}}$. Silvia G. Lage

Av. Enéas de Carvalho Aguiar, 44, Bloco 2, $2^{\circ}$ A, Sala 10

05309-000 São Paulo, SP

Fones: (11) 3069-5697, 3069-5302

Fax: (11) 3069-5397

E-mail: sglage@incor.usp.br

(C)Associação de Medicina Intensiva Brasileira, 2007
RESULTADOS: Os resultados demonstraram homogeneidade dos grupos nos 2 braços (profilático e terapêutico) quanto a sexo, idade, índice de massa corpórea, creatinina sérica e APACHE II. Os valores do anti-fator Xa nos braços profilático e terapêutico demonstraram eficácia adequada dos medicamentos, sem diferença clínica ou estatística entre ambos. A ocorrência de eventos adversos não mostrou diferença clínica ou estatística entre os medicamentos. No braço profilático o ultra-som de membros inferiores acrescentou informações úteis sobre a eficácia dos fármacos estudados.

CONCLUSÕES: A enoxaparina sódica teste (ENOX-T) foi segura e eficaz nos pacientes analisados e equivalente ao medicamento de referência (ENOX-R).

Unitermos: Enoxaparina sódica, Heparina de baixo peso molecular, Profilaxia e terapêutica antitrombótica.

\section{SUMMARY}

BACKGROUND AND OBJECTIVES: Use of low molecular weight heparins $(\mathrm{LMWH})$, either prophylactic or therapeutic, is of most importance in several syndromes and diseases in daily clinical practice. Our objective in this clinical trial was to evaluate safety and efficacy of test sodium enoxaparin (ENOX-T) compared to the reference drug (ENOX-R).

METHODS: We conducted a prospective, randomized, comparative, unicentric and open-labeled trial including patients with either prophylactic or therapeutic antithrombotic indications. A total of 100 patients were enrolled in two branches: prophylactic $(n=50)$ and therapeutic $(n=50)$ and two groups for each branch (group 1: ENOX-R and group 2: ENOX-T). We analyzed clinical and laboratory data in each segment. Anti-factor Xa was measured in three different moments: baseline ( $1^{\text {st }}$ evaluation); $1^{\text {st }}$ or $2^{\text {nd }}$ day ( $2^{\text {nd }}$ evaluation) and $5^{\text {th }}$ to $7^{\text {th }}$ day ( $3^{\text {rd }}$ evaluation). Doppler-sonography of inferior limbs was performed on all patients in prophylactic group on $2^{\text {nd }}$ or $3^{\text {rd }}$ evaluation. 
RESULTS: The data showed that both branches (prophylactic and therapeutic) were homogenous in regard of sex, age, body mass index (BMI), serum creatinine and APACHE II severity score at admission. Anti-factor Xa results, in both prophylactic and therapeutic branches, showed expected efficacy to both drugs, without any clinical or statistical difference between them. Adverse events incurred in both groups in a similar way, without any clinical or statistical difference between them. In prophylactic branch, Doppler-sonography of inferior limbs added useful information on drugs efficacy.

CONCLUSIONS: We conclude that test sodium enoxaparin (ENOX-T) was effective and safe in our patient's cohort and equivalent to reference drug (ENOX-R).

Key Words: Anti-thrombotic prophylaxis and therapy, Low molecular weight heparin, Sodium enoxaparin

\section{INTRODUÇÃO}

O uso de medicação antitrombótica, profilática ou terapêutica, é de extrema importância na prática clínica. $O$ espectro de doenças que merecem indicações precisas de anticoagulação é grande e dispõe de evidências científicas bem consolidadas. Contudo, as alternativas de medicamentos podem ser ampliadas, especialmente no que se refere às opções disponíveis para comercialização no mercado brasileiro.

O racional para a prevenção de trombose venosa profunda (TVP) em pacientes hospitalizados ou com mobilidade física limitada tem sólido respaldo e evidência científica na literatura ${ }^{1-3}$. A relação de pacientes com fatores de risco para a TVP é ampla e prevalência varia de $10 \%$ a $80 \%$ dependendo do tipo de doença ${ }^{4-8}$.

Nas Unidades de Terapia Intensiva o problema é mais heterogêneo e se agrava, pois muitas vezes ocorre intercorrência clínica ou agudização da doença de base, que pode aumentar os riscos de trombose ou sangramento?.

No que se refere à profilaxia de trombose em pacientes clínicos existem seis grandes estudos comparando heparina não fracionada (HNF) ou heparina de baixo peso molecular (HBPM) com placebo ${ }^{10-15}$. Para resumir estes estudos, a comparação entre a não profilaxia e a profilaxia em doses adequadas de heparina não fracionada ou heparina de baixo peso molecular reduziu aproximadamente em $70 \%$ os riscos relativos de trombose, sem aumentar a probabilidade de sangramentos ${ }^{16}$.

Além disso, achou-se conveniente detalhar a importância da dose de HBPM. O estudo mais ilustrativo é o
Medenox realizado para avaliar a profilaxia de eventos trombóticos em pacientes clínicos com a Enoxaparina sódica que estabeleceu a dose de $40 \mathrm{mg}$ uma vez ao dia como eficaz ${ }^{14}$.

No âmbito terapêutico os problemas trombóticos estabelecidos tiveram grande auxílio com o advento das HBPM. Eventos de trombose venosa profunda abaixo da região poplítea, tromboembolismo pulmonar de pequenas proporções e síndromes coronarianas agudas foram incluídos na relação de doenças em que as heparinas de baixo peso molecular estão indicadas ${ }^{17,18}$.

Em particular, as síndromes coronarianas agudas foram motivo de diversos estudos. Considerando-se as diferentes possibilidades de apresentação do quadro agudo, as terapêuticas intervencionistas e a associação de fármacos fibrinolíticos ou antiagregantes plaquetários o tema tem merecido metanálises e diretrizes para que a evidência científica seja a melhor possível. Recentemente em nosso meio foi publicada a "III Diretriz sobre o tratamento do Infarto Agudo do Miocárdio". Quanto à utilização das diferentes heparinas, salientase a indicação da heparina de baixo peso molecular (enoxaparina sódica) na síndrome coronariana aguda sem supradesnivelamento do segmento ST (classe I, evidência $A)^{18}$.

Considerando-se a relevância da aplicabilidade clínica das heparinas de baixo peso molecular, observa-se o empenho na área farmacêutica com a finalidade de obter medicamentos seguros e eficazes, com melhor relação custo/benefício. Este estudo teve por objetivo avaliar a segurança e eficácia de uma enoxaparina sódica teste com a enoxaparina sódica referência em pacientes sob profilaxia e terapêutica antitrombótica.

\section{MÉTODO}

Realizou-se estudo prospectivo, unicêntrico, aleatório e comparativo envolvendo pacientes com indicação de profilaxia ou terapêutica antitrombótica, após a aprovação pelo Comitê de Ética e Pesquisa do Hospital das Clínicas da Faculdade de Medicina da Universidade de São Paulo (CAPPESQ) e obtido a assinatura no Termo de Consentimento Livre e Esclarecido dos pacientes ou responsáveis legais.

No período de maio de 2005 a outubro de 2006 foram incluídos no estudo 100 pacientes internados na Unidade de Terapia Intensiva do Instituto do Coração do Hospital das Clínicas da Faculdade de Medicina da USP, por situações agudas ou descompensação da doença de base. Foram divididos em 2 braços: braço 
profilático (50 pacientes) e braço terapêutico (50 pacientes), cada um dos braços foi dividido em 2 grupos de 25 pacientes: o grupo 1 recebeu enoxaparina sódica de referência (ENOX-R, Clexane ${ }^{\circledR}$, Sanofi - Aventis) e o grupo 2 recebeu enoxaparina sódica, teste (ENOX-T, Cutenox $^{\circledR}$, Instituto Biochimico). A posologia da enoxaparina no braço profilático foi de $40 \mathrm{mg}$, uma vez ao dia e no braço terapêutico de $1 \mathrm{mg} / \mathrm{kg}$ a cada 12 horas, por via subcutânea.

Os critérios de inclusão foram pacientes maiores de 18 anos, obedecendo ao Consenso da Literatura Médica para indicação e uso da enoxaparina sódica no que se refere a profilaxia e terapêutica antitrombótica ${ }^{1,2,14,16-18}$. Os critérios de exclusão foram pacientes com discrasia sanguínea; insuficiência renal (sob procedimento dialítico ou creatinina maior que $2,5 \mathrm{mg} / \mathrm{dL}$ ); sangramento digestivo ativo ou antecedente de sangramento nos últimos três meses; previsão de procedimento invasivo nas 4-6 horas subseqüentes à avaliação inicial; uso concomitante de medicamentos que afetem a hemostasia (fibrinolíticos, heparina não fracionada, anticoagulantes orais); plaquetopenia (abaixo de $100.000 / \mathrm{mm}^{3}$ ); acidente vascular encefálico há 6 meses; insuficiência hepática; hipertensão arterial grave não controlada por medicamentos; retinopatia diabética; neurocirurgia; cirurgia oftálmica recente; gravidez e amamentação.

\section{Protocolo do Estudo}

A avaliação clínica e laboratorial foi registrada em um formulário de registro clínico (FRC) e incluiu três avaliações: $1^{\mathrm{a}}$ avaliação (pré-tratamento), $2^{\mathrm{a}}$ avaliação ( $1^{\circ}$ ou $2^{\circ} \mathrm{dia}$ ) e $3^{\mathrm{a}}$ avaliação ( $5^{\circ}$ ao $7^{\circ} \mathrm{dia}$ ). Foram registrados os dados clínicos selecionados ( $1^{\text {a }}$ avaliação) diagnósticos principal e secundário, idade, sexo, raça, peso, altura, índice de massa corpórea, pressão arterial sistêmica, freqüência cardíaca, temperatura, APACHE II e escala de coma de Glasgow, dados laboratoriais gerais ( $1^{\text {a }}$ avaliação) glicemia, hemograma, contagem de plaquetas, coagulograma completo, bilirrubinas, TGO, TGP, gama-GT, sódio, potássio, uréia e creatinina e dados laboratoriais específicos $\left(1^{\mathrm{a}}, 2^{\mathrm{a}}\right.$ e $3^{\mathrm{a}}$ avaliações) dosagem da atividade heparínica anti-fator Xa cuja coleta foi realizada entre 3 e 5 horas após a injeção por via subcutânea do medicamento. No braço profilático foi realizado ultra-som com Doppler nos membros inferiores na $3^{\mathrm{a}}$ avaliação, isto é, entre $\circ 5^{\circ}$ e $7^{\circ}$ dias de tratamento. Conforme diagnóstico clínico e evolução do caso era possível finalizar o estudo na $2^{\mathrm{a}}$ avaliação. Neste caso realizou-se o ultra-som com Doppler na $2^{\mathrm{a}}$ avaliação, isto é, entre o $1^{\circ}$ ou $2^{\circ}$ dia.

O cronograma de avaliações incluiu a triagem para elegibilidade na $1^{\text {a }}$ Avaliação. Os procedimentos realizados foram: confirmação da elegibilidade, obtenção do consentimento livre e esclarecido por escrito, antes da realização de qualquer procedimento exigido pelo protocolo, obtenção da história clínica completa do paciente, revisão do uso de medicamentos concomitantes, avaliação de sinais vitais (exame físico), inclusive peso e altura, coleta de amostras para os exames laboratoriais (bioquímica sangüínea e hematologia), aleatorização do paciente após a confirmação de que todos os critérios de inclusão foram preenchidos. $\mathrm{Na}$ $2^{\mathrm{a}}$ avaliação $\left(1^{\circ}-2^{\circ}\right.$ dia) incluiu: verificação de eventos adversos desde a $1^{\circ}$ avaliação confirmação de critérios de inclusão e exclusão, coleta de amostras para exames laboratoriais específicos, entrega e registro do medicamento em estudo de acordo com a aleatorização. $\mathrm{Na} 3^{\mathrm{a}}$ avaliação $\left(5^{\circ}-7^{\circ} \mathrm{dia}\right)$ observou-se a existência de eventos adversos desde a $2^{\mathrm{a}}$ avaliação, coleta das amostras para exames laboratoriais específicos, ultra-som Doppler dos membros inferiores (no braço profilático) e encerramento do estudo.

A aleatorização dos grupos de tratamento de ENOX-R e ENOX-T foram na proporção de 1:1 em blocos com alocação igual de tratamentos dentro de cada bloco, sendo que a geração de números foi obtida aleatoriamente. $\mathrm{O}$ acesso a esta ficha ficou restrito ao monitor da pesquisa. As pessoas diretamente envolvidas na condução e/ou análise do estudo não tiveram acesso à alocação ao tratamento até o encerramento da pesquisa

Os pacientes que foram excluídos do estudo após a classificação não foram substituídos. Os pacientes que foram excluídos do estudo antes da classificação foram substituídos, até que o número planejado de pacientes fosse obtido. Foi documentado no FRC o motivo da exclusão de cada paciente. As razões previstas para interrupção do estudo enquadraram-se nas seguintes situações: eventos adversos graves, efeito terapêutico insatisfatório, violação do protocolo, recusa do paciente em participar do estudo e óbito.

\section{Medicamentos do Estudo}

A medicação do estudo ENOX-R e ENOX-T foram armazenadas com acesso limitado, a temperatura ambiente $25^{\circ} \mathrm{C}$, protegida contra luz e umidade. A numeração dos lotes e data de validade foi devidamente anotada no FRC. 


\section{Eventos Adversos}

As informações sobre os eventos adversos foram coletadas e relatadas em folha específica do FRC. A definição usada de eventos adversos é aquela determinada pela Agência Nacional de Vigilância Sanitária (ANVISA). Todos eventos adversos foram relatados ao CEP-HCFMUSP e a ANVISA.

\section{Análise Estatística}

O tamanho mínimo previsto da amostra para esta pesquisa foi de 21 pacientes para cada grupo, calculado a partir do delineamento do projeto que considerava três avaliações por paciente, indicando o uso da Análise de Variância com medidas repetidas em nível significativo de $5 \%$ e poder de teste de $80 \%$

Todas as variáveis foram analisadas descritivamente. Para as variáveis quantitativas foi feita a observação dos valores mínimos e máximos, e do cálculo de médias e desvios-padrão e medianas. Para as variáveis qualitativas calcularam-se freqüências absolutas e relativas. Para a análise da hipótese de igualdade de médias entre os dois grupos utilizou-se o teste $t$ de Student, quando a suposição de normalidade dos dados foi rejeitada utilizou-se o teste não-paramétrico de Mann-Whitney. Para verificar a homogeneidade entre proporções dos dois grupos foi utilizado o teste Qui-quadrado ou o Exato de Fisher. Na comparação da variável anti-fator Xa foi utilizado o teste não paramétrico de Friedman para comparação das avaliações dentro de cada grupo e o teste não paramétrico de Mann-Whitney (com correção de Bonferroni) para comparação entre os grupos em cada momento de avaliação. O nível de significância utilizado para os testes foi de 5\%. Quando da correção de Bonferroni, as diferenças significativas foram consideradas com $\mathrm{p}<0,017$.

\section{RESULTADOS}

Foram incluídos 50 pacientes no braço profilático e 50 no braço terapêutico. Houve exclusão de três pacientes do braço profilático por questões operacionais. Os grupos 1 e 2, isto é, que receberam ENOX-R e ENOX$\mathrm{T}$, respectivamente, em cada braço, foram homogêneos em relação à distribuição de sexo, idade, índice de massa corpórea (IMC), faixa de normalidade de creatinina $(0,6$ a $1,4 \mathrm{mg} / \mathrm{dL})$ e APACHE II no momento da inclusão do estudo (Tabela 1).
Tabela 1 - Distribuição de Sexo, Idade, Índice de Massa Corpórea, Faixa de Normalidade de Creatinina e APACHE II no Momento da Inclusão do Estudo.

\begin{tabular}{|c|c|c|c|c|}
\hline \multirow[t]{2}{*}{ Variáveis } & \multicolumn{2}{|c|}{ Braço Profilático } & \multicolumn{2}{|c|}{ Braço Terapêutico } \\
\hline & $\begin{array}{c}\text { ENOX-R } \\
(\mathrm{n}=24)\end{array}$ & $\begin{array}{l}\text { ENOX-T } \\
(\mathrm{n}=23)\end{array}$ & $\begin{array}{c}\text { ENOX-R } \\
(\mathrm{n}=25)\end{array}$ & $\begin{array}{r}\text { ENOX-T } \\
(n=25)\end{array}$ \\
\hline \multicolumn{5}{|l|}{ Sexo } \\
\hline Masculino & 13 & 16 & 15 & 11 \\
\hline Feminino & 11 & 7 & 10 & 14 \\
\hline Idade (anos) & $64 \pm 14$ & $58 \pm 15$ & $63 \pm 15$ & $62 \pm 15$ \\
\hline $\begin{array}{l}\text { Índice de massa } \\
\text { corpórea }\left(\mathrm{kg} / \mathrm{m}^{2}\right)\end{array}$ & $25 \pm 4$ & $25 \pm 4$ & $25 \pm 4$ & $25 \pm 4$ \\
\hline $\begin{array}{l}\text { Creatinina * (faixa } \\
\text { normal) }\end{array}$ & $79 \%$ & $78 \%$ & $96 \%$ & $92 \%$ \\
\hline APACHE II & $9,8 \pm 3,8$ & $8,5 \pm 5,3$ & $10,7 \pm 4,8$ & $9,5 \pm 3,6$ \\
\hline
\end{tabular}

Não houve diferença estatística dos parâmetros analisados considerando os 2 grupos em cada braço do estudo.(ENOX-R= Clexane $^{\circledR} ; E N O X-T=$ Cutenox $^{\circledR}$ )

* Creatinina acima de $2.5 \mathrm{mg} / \mathrm{dL}$ foi critério de exclusão do estudo e a faixa normal é de 0,6 a $1,4 \mathrm{mg} / \mathrm{dL}$.

\section{Braço Profilático}

O diagnóstico principal incluiu disritmias supraventriculares $(11 \%)$, insuficiência cardíaca/choque cardiogênico (23\%), síndromes coronarianas agudas (23\%), infecção/choque séptico (26\%), outros (17\%).

No que se refere a segurança dos medicamentos observou-se 11 pacientes com eventos adversos no total de 47 analisados (Tabela 2). Nenhum evento relacionado aos medicamentos foi considerado grave e não houve diferença clínica ou estatística entre os grupos. Um evento provavelmente relacionado a ENOX-R foi trombose venosa em membro superior direito na região de cateter venoso e um evento adverso não grave, possivelmente relacionado a ENOX-T foi epistaxe.

Tabela 2 - Total de Eventos Adversos no Grupo Profilático

\begin{tabular}{lccc}
\hline Eventos adversos & ENOX-R & ENOX-T & Total \\
\hline Ausente & $20(80 \%)$ & $19(76 \%)$ & 39 \\
Presente & $5(20 \%)$ & $6(24 \%)$ & 11 \\
Total & 25 & 25 & 50 \\
\hline
\end{tabular}

Os eventos adversos presentes incluem os não relacionados aos medicamentos $(n=9)$ e os possivelmente relacionados a medicação (ENOX-R e ENOX-T, $n=1$ ). Valor do Qui-quadrado $=0,117 ; p=0,733$. (ENOX-R=Clexane ${ }^{\circledR}$; ENOX-T= Cutenox ${ }^{\circledR}$ ).

Quanto à eficácia dos medicamentos os valores do anti-fator Xa foram considerados no pré-tratamento $\left(1^{\mathrm{a}}\right.$ avaliação) e na $2^{\mathrm{a}}$ ou $3^{\mathrm{a}}$ avaliação para cada grupo. Os valores médios do anti-fator Xa respectivamente para a $1^{\text {a }}, 2^{\mathrm{a}}$ e $3^{\mathrm{a}}$ avaliação no que se refere aos medicamentos em estudo foram: a) ENOX-R: 0,02 $\pm 0,04 \mathrm{UI} / \mathrm{mL}$; $0,34 \pm 0,15 \mathrm{Ul} / \mathrm{mL} ; 0,37 \pm 0,15 \mathrm{Ul} / \mathrm{mL} ;$ b) ENOX-T: 0,02 $\pm 0,06 \mathrm{UI} / \mathrm{mL} ; 0,34 \pm 0,16 \mathrm{UI} / \mathrm{mL} ; 0,41 \pm 0,17 \mathrm{Ul} / \mathrm{mL}$. A 
representação gráfica dos dados e valores estatísticos encontra-se na figura 1.

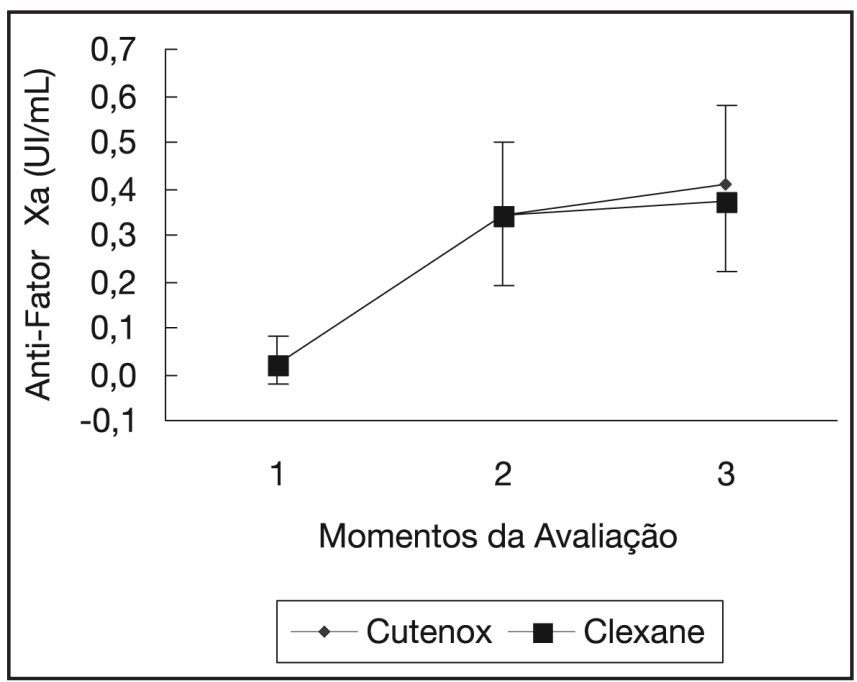

Figura 1 - Valores do Anti-Fator Xa do Braço Profilático nos Pacientes do Grupo 1 (ENOX-R, Clexane ${ }^{\circledR}$ ) e grupo 2 (ENOX-T, Cutenox ${ }^{\circledR}$ nas Três Avaliações.

O basal (1) difere das avaliações $2(p<0,001)$ e $3(p<0,001)$ para ambos medicamentos. A comparação entre os grupos não apresenta diferença estatística (avaliação 1; $p=0,613$ ); (avaliação 2; $p=0,890)$; (avaliação 3; $p=0,765$ ).

O ultra-som Doppler de membros inferiores auxiliou a documentar a eficácia da medicação comprovando ausência de TVP nos pacientes estudados no braço profilático. Dois pacientes demonstraram TVP após o estudo, um ENOX-R e outro ENOX-T, nos quais não havia suspeita clínica prévia de TVP, sua confirmação só foi realizada no término do estudo. Ambos pacientes apresentavam valores do anti-fator $\mathrm{Xa}$ adequados para a profilaxia antitrombótica.

\section{Braço Terapêutico}

O diagnóstico principal incluiu disritmias supraventriculares (29\%); insuficiência cardíaca/choque cardiogênico (21\%); síndromes coronarianas agudas (4\%); TEP/TVP/trombo intracavitário (29\%); hipertensão pulmonar (8,5\%); outros $(8,5 \%)$.

No que se refere a segurança dos medicamentos observou-se 11 pacientes com eventos adversos no total de 50 analisados (Tabela 3). Os eventos adversos, possivelmente relacionados aos medicamentos, foram sangramento de cateter venoso central, sangramento de cânula orotraqueal, sangramento local em escoriação cutânea, hematoma no local da administração do fármaco, epistaxe/hemoptise para ENOX-R (5) e hemoptise, escarro hemoptoico e hematúria para ENOX-T (3).
Tabela 3 - Total de Eventos Adversos no Grupo Terapêutico

\begin{tabular}{lccc}
\hline Eventos adversos & ENOX-R & ENOX-T & Total \\
\hline Ausente & $19(76 \%)$ & $20(80 \%)$ & 39 \\
Presente & $6(24 \%)$ & $5(20 \%)$ & 11 \\
Total & 25 & 25 & 50 \\
\hline
\end{tabular}

Os eventos adversos presentes incluem os não relacionados aos medicamentos $(n=3)$ e os possivelmente relacionados a medicação (ENOX-R, $n=5$ e ENOX-T, $\mathrm{n}=3$ ).

Valor do Qui-quadrado $=0,117 ; p=0,733$. $\left(\right.$ ENOX-R $=$ Clexane $^{\oplus ;}$ ENOX-T $=$ Cutenox ${ }^{\circledR}$ ).

Quanto à eficácia dos medicamentos os valores do anti-fator Xa foram considerados no pré-tratamento $\left(1^{\mathrm{a}}\right.$ avaliação) e na $2^{\mathrm{a}}$ ou $3^{\mathrm{a}}$ avaliação para cada grupo. Os valores médios do anti-fator Xa respectivamente para a $1^{\mathrm{a}}, 2^{\mathrm{a}}$ e $3^{\mathrm{a}}$ avaliações no que se refere aos medicamentos em estudo foram: a) ENOX-R: 0,05 $\pm 0,09 \mathrm{UI} / \mathrm{mL}$; $0,43 \pm 0,15 \mathrm{Ul} / \mathrm{mL} ; 0,63 \pm 0,18 \mathrm{Ul} / \mathrm{mL}$ e b) ENOX-T: 0,08 $\pm 0,20 \mathrm{Ul} / \mathrm{mL} ; 0,48 \pm 0,18 \mathrm{Ul} / \mathrm{mL} ; 0,79 \pm 0,28 \mathrm{Ul} / \mathrm{mL}$. A representação gráfica dos dados e valores estatísticos encontra-se na figura 2.

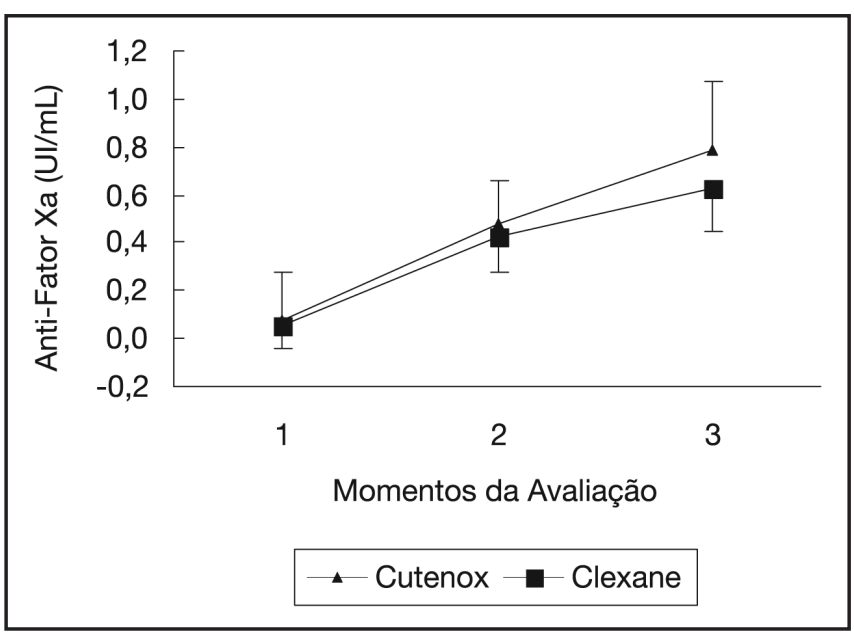

Figura 2 - Valores do Anti-Fator Xa do Braço Terapêutico nos Pacientes do Grupo 1 (ENOX-R, Clexane ${ }^{\circledR}$ ) e grupo 2 (ENOX-T, Cutenox ${ }^{\circledR}$ ) nas Três Avaliações.

O basal (1) difere das avaliações $2(p<0,005)$ e $3(p<0,001)$ para ambos medicamentos. A comparação entre os grupos não apresenta diferença estatística. (avaliação 1; $p=0,913$ ); (avaliação $2=0,473$ ); (avaliação 3; $p=0,027$, NS com a correção de Bonferroni)

\section{DISCUSSÃO}

O fracionamento ou despolimerização da heparina de origem natural resulta em produto denominado HBPM. As características químicas resultantes do fracionamento são de grande importância na obtenção do efeito antitrombótico desejado na clínica.

Por definição as HBPM são sais de glicosaminoglicanos sulfatados, com peso molecular inferior a 8000 daltons (60\% da molécula) e com diferente estrutura 
química na extremidade da cadeia de polissacarídeo (redutora ou não redutora).

A enoxaparina sódica é uma heparina com peso molecular entre 3.500 a 5.500 (valor médio 4.500 daltons). O valor relativo do peso molecular da cadeia inferior a 2.000 daltons varia entre $12 \%$ e $20 \%$ e do peso molecular de 2.000 até 8.000 varia entre $68 \%$ e $88 \%$. O grau de sulfatação é cerca de 2 por unidade dissacarídea. A potência não é menor que 99 UI e não superior a 125 UI de atividade anti-fator Xa por miligrama, calculada com referência à substância seca.

O medicamento de referência da enoxaparina sódica no Brasil é a ENOX-R (Clexane $\left.{ }^{\circledR}\right)$. O medicamento ENOX-T (Cutenox $\left.{ }^{\circledR}\right)$, cujo principio ativo também é a enoxaparina sódica, foi desenvolvido na Índia, pela empresa Gland Pharma e é comercializado no Brasil pelo Instituto BioChimico Indústria Farmacêutica Ltda. Os ensaios in vitro comparativos entre os dois produtos realizados no centro médico da Universidade de Loyola, Chicago, Illinois, USA, demonstraram a equivalência farmacêutica entre ambos e que o ENOX-T cumpre as especificações exigidas.

Desta forma, o ENOX-T já obteve o registro no Ministério da Saúde ( $\left.n^{\circ} 1.0063 .0188\right)$ embora sua equivalência clínica em âmbito nacional ainda não tenha sido avaliada, considerando-se as indicações para uso já aprovadas para o ENOX-R Reg. MS n ${ }^{0} 1.1300 .0276{ }^{19}$.

Neste estudo, os resultados demonstraram que os medicamentos citados foram equivalentes no que se refere a segurança e eficácia para aplicação clínica. Os pacientes incluídos para profilaxia e terapêutica antitrombótica foram em número adequado e distribuição homogênea entre os grupos analisados.

É importante salientar que a dosagem do anti-fator Xa para monitoramento da eficácia da HBPM é recente e especialmente pouco estudada em pacientes gravemente enfermos. Grande atenção tem sido dada aos pacientes com insuficiência renal (IR), tendo em vista o potencial risco de acúmulo do fármaco ${ }^{20-23}$. Apesar da pouca evidência científica envolvendo o tema, a monitorização com a dosagem do anti-fator Xa tem sido recomendada para dois grupos: pacientes com insuficiência renal avançada (definida por depuração de creatinina menor que 30 $\mathrm{mL} / \mathrm{min}$ ) e pacientes obesos ${ }^{24}$.

Outras situações comumente encontradas em pacientes gravemente enfermos, tais como, o baixo débito cardíaco e o edema do tecido subcutâneo - potencialmente podem influenciar na biodisponibilidade da HBPM. A hipótese de que a diminuição da perfusão tecidual poderia interferir na absorção da HBPM, tendo em vista que o fármaco é administrado por via subcutânea, ainda não foi adequadamente investigada. Em relação ao potencial prejuízo da absorção do fármaco devido ao edema do tecido subcutâneo, um estudo recente realizado com pacientes gravemente enfermos, mostrou que a presença de edema subcutâneo não interfere na atividade da HBPM medida através da dosagem do anti-fator Xa ${ }^{25}$. Além deste, poucos estudos utilizaram a dosagem sérica do antifator Xa para avaliação da eficácia da profilaxia em pacientes gravemente enfermos ${ }^{26,27}$. Dados recentes de literatura sugerem que a monitorização do uso da HBPM com a dosagem do anti-fator Xa em pacientes críticos parece ser útil, sobretudo naqueles com baixo debito cardíaco ${ }^{28}$.

No Brasil, um esforço conjunto de diversas associações médicas brasileiras (Academia Brasileira de Neurologia, Associação de Medicina Intensiva Brasileira, Federação Brasileira das Associações de Ginecologia e Obstetrícia, Sociedade Brasileira de Angiologia e de Cirurgia Vascular, Sociedade Brasileira de Cancerologia, Sociedade Brasileira de Cardiologia, Sociedade Brasileira de Clínica Médica, Sociedade Brasileira de Geriatria e Gerontologia, Sociedade Brasileira de Hematologia e Hemoterapia, Sociedade Brasileira de Pneumologia e Tisiologia e Sociedade Brasileira de Reumatologia) sob os auspícios da Associação Médica Brasileira e do Conselho Federal de Medicina integrou ao Projeto Diretrizes os capítulos sobre Tromboembolismo Venoso: Profilaxia em Pacientes Clínicos ${ }^{29}$. A normatização e consolidação do uso adequado das HBPM é fundamental, considerando a ampla aplicabilidade, eficácia do medicamento e custo/benefício na sua indicação.

Este trabalho evidencia que a dosagem do anti-fator Xa foi essencial para controle da eficácia do medicamento e abre perspectivas para análise da dosagem, a partir do $7^{\circ}$ dia, no que se refere ao controle evolutivo de pacientes individualizados, especialmente naqueles com complicações clínicas e em estado crítico.

Neste primeiro estudo brasileiro que comparou a ENOX-R e a ENOX-T concluiu-se que os medicamentos analisados foram equivalentes em segurança e eficácia para pacientes com indicação de profilaxia e terapêutica antitrombótica. Desta forma, acrescenta informações com embasamento científico no que se refere ao uso de medicamentos extremamente úteis na prática clínica. 


\section{AGRADECIMENTOS}

Nossos agradecimentos a Creusa dal Bó pela competente análise estatística, a farmacêutica Carla Adriana Miyashiro pela organização e supervisão do projeto, a Margarete Bueno pela eficiente assistência em todas as etapas da pesquisa, a Mônica Julião, Dr. Pedro Garbes Netto e Christoph Gross pelo inestimável apoio logístico na realização deste estudo.

\section{REFERÊNCIAS}

01. Second Thromboembolic Risk Factors (THRiFT II) Consensus Group. Risk of and prophylaxis for venous thromboembolism in hospital patients. Phlebology, 1998;13:87-97.

02. Geerts WH, Heit JA, Clagett GP et al - Prevention of venous thromboembolism. Chest, 2001;119:(Suppl1):132S-175S.

03. Sullivan SD, Kahn SR, Davidson BL et al - Measuring the outcomes and pharmacoeconomic consequences of venous thromboembolism prophylaxis in major orthopaedic surgery. Pharmacoeconomics, 2003;21:477-496.

04. Anderson FA, Wheeler HB, Goldberg RJ et al - The prevalence of risk factors for venous thromboembolism among hospital patients. Arch Intern Med, 1992;152:1660-1664.

05. Rosendaal FR - Risk factors for venous thrombotic disease. Thromb Haemost, 1999;82:610-619.

06. Kearon C, Salzman EW, Hirsh J - Epidemiology, Pathogenesis, and Natural History of Venous Thrombosis, em: Colman RW, Hirsh J, Marder VJ, et al - Hemostasis and Thrombosis: Basic Principles and Clinical Practice. $4^{\text {th }}$ Ed, Philadelphia, PA: JB Lippincott, 2001;1153-1177.

07. Heit JA, O'Fallon WM, Petterson TM et al - Relative impact of risk factors for deep vein thrombosis and pulmonary embolism: a population-based study. Arch Intern Med, 2002;162:1245-1248.

08. Anderson FA Jr, Spencer FA - Risk factors for venous thromboembolism. Circulation, 2003;107:(Suppl23):19-116.

09. Rocha AT, Tapson VF - Venous thromboembolism in intensive care patients. Clin Chest Med, 2003;24:103-122.

10. Gallus AS, Hirsh J, Tuttle RJ et al - Small subcutaneous doses of heparin in prevention of venous thrombosis. N Engl J Med 1973;288:545-551.

11. Belch JJ, Lowe GD, Ward AG et al - Prevention of deep vein thrombosis in medical patients by low-dose heparin. Scott Med J, 1981;26:115117.

12. Cade JF - High risk of the critically ill for venous thromboembolism. Crit
Care Med, 1982;10:448-450.

13. Dahan $\mathrm{R}$, Houlbert $\mathrm{D}$, Caulin $\mathrm{C}$ et al - Prevention of deep vein thrombosis in elderly medical in-patients by a low molecular weight heparin: a randomized double-blind trial. Haemostasis, 1986;16:159-164.

14. Samama MM, Cohen AT, Darmon JY et al - A comparison of enoxaparin with placebo for the prevention of venous thromboembolism in acutely ill medical patients. N Engl J Med, 1999;341:793-800.

15. Leizorovicz A, Cohen AT, Turpie AG et al - Randomized, placebo controlled trial of dalteparin for the prevention of venous thromboembolism in acutely ill medical patients: Circulation, 2004,110:874-879.

16. Geerts WH, Pineo GF, Heit JA et al - Prevention of venous thromboembolism: the Seventh ACCP Conference on Antithrombotic and Thrombolytic Therapy. Chest, 2004;126: (Suppl3):338S-400S.

17. Kopel L, Carvalho RT, Lage SG - Tromboembolia Pulmonar, em: Ramires JAF, Oliveira AS Cardiogeriatria. São Paulo, Roca, 2004;230-245.

18. Arquivos Brasileiros de Cardiologia, III Diretriz sobre tratamento do infarto agudo do miocárdio. 2004;83:(SupplIV):86.

19. Dicionário de Especialidades Farmacêuticas: DEF $2002 / 03-31^{\text {a }}$ Ed, Rio de Janeiro: Publicações Científicas, 2002;334-337.

20. Rabbat CG, Cook DJ, Crowther MA - Dalteparin thromboprophylaxis for critically ill medical-surgical patients with renal insufficiency. J Crit Care, 2005;20:357-363

21. Nagge J, Crowther M, Hirsh $\mathrm{J}$ - Is impaired renal function a contraindication to the use of low-molecular-weight heparin? Arch Intern Med 2002;162:2605-2609.

22. Goudable C, Saivin S, Houin G et al - Pharmacokinetics of a low molecular weight heparin (Fraxiparine) in various stages of chronic renal failure. Nephron, 1991;59:543-545

23. Hory $\mathrm{B}$, Claudet $\mathrm{MH}$, Magnette $\mathrm{J}$ et al - Pharmacokinetics of a very low molecular weight heparin in chronic renal failure. Thromb Res, 1991;63:311-317.

24. Johnson PN, Smith KM - Low-molecular-weight heparin use in special populations. Orthopedics 2004;27:1245-1248.

25. Kapoor M, Kupfer YY, Tessler S - Subcutaneous heparin prophylaxis significantly reduces the incidence of venous thromboembolic events in the critically ill. Crit Care Med, 1999;27:(Suppl):A69.

26. Cook DJ, Rocker G, Meade M et al - Prophylaxis of Thromboembolism in Critical Care (PROTECT) Trial: a pilot study. J Crit Care, 2005;20:364372.

27. Lage S, Carvalho R, Kopel L et al - Use of anti-Xa factor titration as efficacy marker of enoxaparin in critically ill patients. Crit Care. 2006;10:(Suppl 1):S67.

28. Rommers MK, Van der Lely N, Egberts TC et al - Anti-Xa activity after subcutaneous administration of dalteparin in ICU patients with and without subcutaneous oedema: a pilot study. Crit Care 2006;10:R93.

29. Projeto Diretrizes. Tromboembolismo Venoso: Profilaxia em Pacientes Clínicos - Partes I, II e III. http://www.projetodiretrizes.org.br/novas_diretrizes.php, acessado em 09 de novembro de 2006. 